DOI: $10.15393 /$ j3.art.2019.6150

UDC 517.5, 517.98, 512.5

K. Porselvi, B. Elavarasan

\title{
ON HYBRID INTERIOR IDEALS IN SEMIGROUPS
}

\begin{abstract}
In this paper, we introduce the notion of hybrid interior ideals and hybrid characteristic interior ideals of a semigroup. We obtain some equivalent conditions for a hybrid structure to be a hybrid interior ideal of a semigroup. Also, we show that hybrid interior ideals and hybrid ideals coincide for a regular semigroup and a intra-regular semigroup.
\end{abstract}

Key words: Semigroup, ideals, regular, simple, hybrid structure, hybrid interior ideal

2010 Mathematical Subject Classification: 20M12, 20M17, $16 Y 60$

1. Introduction. A semigroup is an algebraic structure consisting of a non-empty set $S$ together with an associative binary operation [4]. Semigroups are important in many areas of mathematics and the concepts of semigroups have been studied by several researchers (see [2] and [5]).

Let $S$ be a semigroup. Let $A$ and $B$ be subsets of $S$. Then the multiplication of $A$ and $B$ is defined as $A B=\{a b: a \in A$ and $b \in B\}$. Let $A$ be a non-empty subset of $S$; $A$ is called a subsemigroup of $S$ if $A^{2} \subseteq A$. A subsemigroup $A$ of $S$ is called a left (resp., right) ideal of $S$ if $S A \subseteq A$ (resp., $A S \subseteq A$ ). If $A$ is both a left and a right ideal of $S$, then $A$ is called a two-sided ideal or ideal of $S$. It can easily be verified that for any $a \in S, L(a)=\{a \cup S a\}$ (resp., $R(a)=\{a \cup a S\}$ ) is a left (resp., right) ideal of $\mathrm{S}$ generated by $a$ in $S$. A semigroup $S$ is called regular if for each $a \in S$ there exists an element $x$ in $S$ such that $a=a x a$. A semigroup $S$ is regular if every element of $S$ is regular [11]. A semigroup $S$ is called intra-regular if for each $a \in S$ there exist $x, y \in S$, such that $a=x a^{2} y$ [11]. Let $S$ be a semigroup. A subsemigroup $A$ of $S$ is called an interior ideal of $S$ if $S A S \subseteq A$.

(C) Petrozavodsk State University, 2019 
In 1965, Zadeh [13] introduced the concept of fuzzy subsets and studied their properties on the parallel line to the set theory. In 1967, Rosenfeld [12] defined the notion of a fuzzy subgroup and its structure was investigated. Later, several authors have been pursued the study of fuzzy algebraic structures in many directions, such as groups, rings, semigroups, ordered semigroups and so on (see [6], [7], [8], and [10]). In 2017, Anis and et al. have introduced the notions of hybrid sub-semigroups and hybrid left (resp., right) ideals in semigroups and obtained several properties [1].

In [9], Kuroki introduced the notion of fuzzy semiprimality in a semigroup, which is an extension of semiprimality in it, and characterized a semigroup that is a semilattice of simple semigroups in terms of fuzzy semiprimality. Following [9], Hong and et al. studied fuzzy interior ideals and discussed fuzzy characteristic interior ideal of semigroup [3].

In this paper, we introduce the notion of hybrid interior ideal and hybrid characteristic interior ideal of semigroups and investigate some of their properties for semigroups and regular semigroups.

2. Hybrid structures in semigroups. We now start this section with basic definitions of a hybrid structure of semigroup that are needed in this paper.

Definition 1. [1] Let $I$ be the unit interval and $\mathcal{P}(U)$ denote the power set of an initial universal set $U$. A hybrid structure in $S$ over $U$ is defined to be a mapping $\tilde{f}_{\lambda}:=(\tilde{f}, \lambda): S \rightarrow \mathcal{P}(U) \times I, x \mapsto(\tilde{f}(x), \lambda(x))$, where $\tilde{f}: S \rightarrow \mathcal{P}(U)$ and $\lambda: S \rightarrow I$ are mappings.

Let us denote by $H(S)$ the set of all hybrid structures in $S$ over $U$. We define an order $\ll$ in $H(S)$ as follows: For all $\tilde{f}_{\lambda}, \tilde{g}_{\gamma} \in H(S), \tilde{f}_{\lambda} \ll \tilde{g}_{\gamma}$ if and only if $\tilde{f} \subseteq \tilde{g}, \lambda \succeq \gamma$, where $\tilde{f} \subseteq \tilde{g}$ means that $\tilde{f}(x) \subseteq \tilde{g}(x)$ and $\lambda \succeq \gamma$ means that $\lambda(x) \geqslant \gamma(x)$ for all $x \in S$. Note that $(H(S), \ll)$ is a poset.

Definition 2. [1] Let $S$ be a semigroup. A hybrid structure $\tilde{f}_{\lambda}$ in $S$ is called a hybrid subsemigroup of $S$ over $U$ if $\tilde{f}(x y) \supseteq \tilde{f}(x) \cap \tilde{f}(y)$ and $\lambda(x y) \leqslant \bigvee\{\lambda(x), \lambda(y)\}$ for all $x, y \in S$.

Definition 3. [1] Let $S$ be a semigroup. A hybrid structure $\tilde{f}_{\lambda}$ in $S$ over $U$ is called a hybrid left (resp., right) ideal of $S$ over $U$ if

(i) $\tilde{f}(x y) \supseteq \tilde{f}(y)$ (resp., $\tilde{f}(x y) \supseteq \tilde{f}(x))$,

(ii) $\lambda(x y) \leqslant \lambda(y)$ (resp., $\lambda(x y) \leqslant \lambda(x)$ ) for all $x, y \in S$.

A hybrid structure $\tilde{f}_{\lambda}$ is called a hybrid ideal of $S$ if it is both a hybrid left ideal and a hybrid right ideal of $S$ over $U$. 
In [9], Kuroki introduced the notion of fuzzy semiprimality in a semigroup, which is an extension of semiprimality in it, and characterizaed a semigroup that is a semilattice of simple semigroups in terms of fuzzy semiprimality. We define the notion of hybrid interior ideal in $S$ over $U$ as follows:

Definition 4. Let $S$ be a semigroup. A hybrid subsemigroup $\tilde{f}_{\lambda}$ in $S$ over $U$ is called a hybrid interior ideal of $S$ over $U$ if

(i) $\tilde{f}(x a y) \supseteq \tilde{f}(a)$,

(ii) $\lambda(x a y) \leqslant \lambda(a)$ for all $a, x, y \in S$.

It is easy to verify that every hybrid ideal of $S$ is a hybrid interior ideal of $S$, but the converse need not be true, as can be seen by the following example.

Example 1. Let $S=\{0, a, b, c\}$ be a semigroup with the following Cayley table:

\begin{tabular}{c|cccc}
$\cdot$ & 0 & $a$ & $b$ & $c$ \\
\hline 0 & 0 & 0 & 0 & 0 \\
$a$ & 0 & 0 & $a$ & $a$ \\
$b$ & 0 & 0 & $b$ & $b$ \\
$c$ & 0 & 0 & $c$ & $c$
\end{tabular}

Let $\tilde{f}_{\lambda}$ be a hybrid structure in $S$ over $U=[0,1]$ defined by $\tilde{f}(0)=[0,0.8] ; \tilde{f}(a)=[0,0.6] ; \tilde{f}(b)=[0,0.5] ; \tilde{f}(c)=[0,0.3]$ and $\lambda$ be any constant mapping from $S$ to $I$. Then $\tilde{f}_{\lambda}$ is a hybrid interior ideal of $S$ over $U$, but it is not a hybrid ideal of $S$ over $U$ as $\tilde{f}(c b) \nsupseteq \tilde{f}(b)$.

Let $A$ be a non-empty subset of $S$. Then the characteristic hybrid structure in $S$ over $U$ is denoted by $\chi_{A}\left(\tilde{f}_{\lambda}\right)=\left(\chi_{A}(\tilde{f}), \chi_{A}(\lambda)\right)$, where

$$
\chi_{A}(\tilde{f}): S \rightarrow \mathcal{P}(U), \quad x \mapsto\left\{\begin{array}{lc}
U & \text { if } x \in A, \\
\phi & \text { otherwise }
\end{array}\right.
$$

and

$$
\chi_{A}(\lambda): S \rightarrow I, \quad x \mapsto \begin{cases}0 & \text { if } x \in A, \\ 1 & \text { otherwise }\end{cases}
$$

for all $x \in S$.

Definition 5. [1] Let $\tilde{f}_{\lambda}$ be a hybrid structure in $S$ over $U$. Then, for $\alpha \in \mathcal{P}(U)$ and $t \in[0,1]$, the set $\tilde{f}_{\lambda}[\alpha, t]:=\{x \in X \mid \tilde{f}(x) \supseteq \alpha, \lambda(x) \leqslant t\}$ is called the $[\alpha, t]$-hybrid cut in $S$ over $U$. 
3. Hybrid interior ideals in semigroups. In this section, we discuss hybrid interior ideals and hybrid characteristic interior ideals of a semigroup and now we present a useful theorem.

Theorem 1. [1] Let $A$ be a non-empty subset of $S$. Then $A$ is a subsemigroup of $S$ if and only if the characteristic hybrid structure $\chi_{A}\left(\tilde{f}_{\lambda}\right)$ is a hybrid subsemigroup of $S$.

Theorem 2. Let $A$ be a non-empty subset of $S$. Then $A$ is an interior ideal of $S$ if and only if the characteristic hybrid structure $\chi_{A}\left(\tilde{f}_{\lambda}\right)$ is a hybrid interior ideal of $S$.

Proof. Assume that $A$ is an interior ideal of $S$ and $a, x, y \in S$. Then, by Theorem $1, \chi_{A}\left(\tilde{f}_{\lambda}\right)$ is a hybrid subsemigroup of $S$.

If $a \in A$, then $\chi_{A}(\tilde{f})(a)=U$ and $\chi_{A}(\lambda)(a)=0$. Since $A$ is an interior ideal of $S$, we have $x a y \in A$. So, $\chi_{A}(\tilde{f})(x a y)=U=\chi_{A}(\tilde{f})(a)$ and $\chi_{A}(\lambda)(x a y)=0 \leqslant \chi_{A}(\lambda)(a)$. If $a \notin A$, then $\chi_{A}(\tilde{f})(a)=\phi \subseteq \chi_{A}(\tilde{f})(x a y)$ and $\chi_{A}(\lambda)(x a y) \leqslant \chi_{A}(\lambda)(a)=1$. Therefore, $\chi_{A}\left(\tilde{f}_{\lambda}\right)$ is a hybrid interior ideal of $S$.

Conversely, assume that $\chi_{A}\left(\tilde{f}_{\lambda}\right)$ is a hybrid interior ideal of $S$. Then, by Theorem 1, $A$ is a subsemigroup of $S$. Let $x, y \in S$ and $a \in A$. Then $\chi_{A}(\tilde{f})(x a y) \supseteq \chi_{A}(\tilde{f})(a)=U$ and $\chi_{A}(\lambda)(x a y) \leqslant \chi_{A}(\lambda)(a)=0$, which imply $x a y \in A$. Thus, $S A S \subseteq A$ and, hence, $A$ is an interior ideal of $S$.

The following theorems show that a hybrid ideal and a hybrid interior ideal coincide for a regular semigroup and for an intra-regular semigroup.

Theorem 3. Let $S$ be a regular semigroup. If $\tilde{f}_{\lambda}$ is a hybrid interior ideal of $S$, then $\tilde{f}_{\lambda}$ is a hybrid ideal of $S$.

Proof. Let $x, y \in S$. Then $\tilde{f}(x y) \supseteq \tilde{f}(x)$ and $\lambda(x y) \leqslant \lambda(x)$. Indeed: Since $S$ is regular and $x \in S$, there exists $z \in S$ such that $x=x z x$. Now, $x y=(x z) x y$. Since $\tilde{f}_{\lambda}$ is a hybrid interior ideal of $S$, we have $\tilde{f}(x y)=\tilde{f}((x z) x y) \supseteq \tilde{f}(x)$ and $\lambda(x y)=\lambda((x z) x y) \leqslant \lambda(x)$, so $\tilde{f}_{\lambda}$ is a hybrid right ideal of $S$. Similarly, we can prove that $\tilde{f}_{\lambda}$ is a hybrid left ideal of $S$. Therefore, $\tilde{f}_{\lambda}$ is a hybrid ideal of $S$.

Theorem 4. Let $S$ be an intra-regular semigroup. If $\tilde{f}_{\lambda}$ is a hybrid interior ideal of $S$, then $\tilde{f}_{\lambda}$ is a hybrid ideal of $S$.

Proof. Let $x, y \in S$. Then $\tilde{f}(x y) \supseteq \tilde{f}(x)$ and $\lambda(x y) \leqslant \lambda(x)$. Indeed: Since $S$ is intra-regular and $x \in S$, there exist $a, b \in S$ such that $x=$ $=a x^{2} b$. Now $x y=a x^{2} b y$. Since $\tilde{f}_{\lambda}$ is a hybrid interior ideal of $S$, we have 
$\tilde{f}(x y)=\tilde{f}\left(a x^{2} b y\right) \supseteq \tilde{f}(x)$ and $\lambda(x y)=\lambda\left(a x^{2} b y\right) \leqslant \lambda(\underset{\tilde{f}}{\lambda})$ and so $\tilde{f}_{\lambda}$ is a hybrid right ideal of $S$. Similarly, we can prove that $\tilde{f}_{\lambda}$ is a hybrid left ideal of $S$. Therefore, $\tilde{f}_{\lambda}$ is a hybrid ideal of $S$.

Theorem 5. Let $S$ be a semigroup. If $\tilde{f}_{\lambda}$ is a hybrid interior ideal of $S$, then $[\alpha, t]$-hybrid cut $\tilde{f}_{\lambda}[\alpha, t]$ of $S$ is an interior ideal of $S$ for every $\alpha \in \mathcal{P}(U)$ and $t \in[0,1]$, provided that $\tilde{f}_{\lambda}[\alpha, t] \neq \phi$.

Proof. Let $\alpha \in \mathcal{P}(U)$ and $t \in[0,1]$ with $\tilde{f}_{\lambda}[\alpha, t] \neq \phi$. Then, for any $x, y \in \tilde{f}_{\lambda}[\alpha, t]$, we have $\tilde{f}(x) \supseteq \alpha, \tilde{f}(y) \supseteq \alpha, \lambda(x) \leqslant t$ and $\lambda(y) \leqslant t$, which implies $\tilde{f}(x y) \supseteq \tilde{f}(x) \cap \tilde{f}(y) \supseteq \alpha$ and $\lambda(x y) \leqslant \bigvee\{\lambda(x), \lambda(y)\} \leqslant t$. So, $x y \in \tilde{f}_{\lambda}[\alpha, t]$. Therefore, $\tilde{f}_{\lambda}[\alpha, t]$ is a subsemigroup of $S$. Let $x, y \in S$ and $a \in \tilde{f}_{\lambda}[\alpha, t]$. Then $\tilde{f}(x a y) \supseteq \tilde{f}(a) \supseteq \alpha$ and $\lambda(x a y) \leqslant \lambda(a) \leqslant t$. Thus, $x a y \in \tilde{f}_{\lambda}[\alpha, t]$ and, hence, $\tilde{f}_{\lambda}[\alpha, t]$ is an interior ideal of $S$.

Theorem 6. Let $S$ be a semigroup. If $J$ is an interior ideal of $S$, then for every $t \in(0,1)$ there exists a hybrid interior ideal $\tilde{f}_{\lambda}$ of $S$ such that $[\alpha, t]$-hybrid cut $\tilde{f}_{\lambda}[\alpha, t]=J$, where $\alpha \in \mathcal{P}(U)$.

Proof. Let $J$ be an interior ideal of $S$ and let $\tilde{f}_{\lambda}$ be a hybrid structure of $S$ defined by

$$
\tilde{f}(x)=\left\{\begin{array}{ll}
\alpha & \text { if } x \in J, \\
\phi & \text { if } x \notin J
\end{array} \quad \text { and } \quad \lambda(x)= \begin{cases}0 & \text { if } x \in J, \\
t & \text { if } x \notin J\end{cases}\right.
$$

and $\tilde{f}_{\lambda}[\alpha, t]$ be a $[\alpha, t]$-hybrid cut.

Let $x, y \in S$. If $x, y \in J$, then $x y \in J$. So, $\tilde{f}(x y)=\alpha=\tilde{f}(x) \cap \tilde{f}(y)$ and $\lambda(x y)=0=\bigvee\{\lambda(x), \lambda(y)\}$. If $x, y \notin J$, then $\tilde{f}(x)=\phi=\tilde{f}(y)$ and $\lambda(x)=t=\lambda(y)$. So, $\tilde{f}(x y) \supseteq \tilde{f}(x) \cap \tilde{f}(y)$ and $\lambda(x y) \leqslant \bigvee\{\lambda(x), \lambda(y)\}$. If $x \notin J$ or $y \notin J$, then $\tilde{f}(x y) \supseteq \tilde{f}(x) \cap \tilde{f}(y)$ and $\lambda(x y) \leqslant \bigvee\{\lambda(x), \lambda(y)\}$. Thus, $\tilde{f}_{\lambda}$ is a hybrid subsemigroup of $S$.

Now, let $a, x, y \in S$. If $a \in J$, then $x a y \in J$. So, $\tilde{f}(x a y)=\alpha=\tilde{f}(a)$ and $\lambda(x a y)=0=\lambda(a)$. If $a \notin J$, then $\tilde{f}(a)=\phi$ and $\lambda(a)=t$, and so $\tilde{f}(x a y) \supseteq \tilde{f}(a)$ and $\lambda(x a y) \leqslant \lambda(a)$. Therefore, $\tilde{f}_{\lambda}$ is a hybrid interior ideal of $S$. By Theorem $5, \tilde{f}_{\lambda}[\alpha, t]$ is an interior ideal of $S$.

If $x \in J$, then $\tilde{f}(x)=\alpha$ and $\lambda(x)=0$, which implies $\tilde{f}(x) \supseteq \alpha$ and $\lambda(x) \leqslant t$. So, $x \in \tilde{f}_{\lambda}[\alpha, t]$ and, therefore, $J \subseteq \tilde{f}_{\lambda}[\alpha, t]$. Suppose that $x \in \tilde{f}_{\lambda}[\alpha, t]$. Then $\tilde{f}(x) \supseteq \alpha$ and $\lambda(x) \leqslant t$. If $\lambda(x)<t$, then $x \in J$. If $\tilde{f}(x) \supseteq \alpha$ and $\lambda(x)=t$, then $x \in J$ and $x \notin J$ : a contradiction. Thus, $\tilde{f}_{\lambda}[\alpha, t]=J$.

Notation 1. For any hybrid structure $\tilde{f}_{\lambda}$ of $S$, we define 


$$
\operatorname{Im}\left(\tilde{f}_{\lambda}\right)=(\operatorname{Im}(\tilde{f}), \operatorname{Im}(\lambda)) .
$$

Theorem 7. Let $\tilde{f}_{\lambda}$ be a hybrid left ideal (resp., hybrid right ideal, hybrid ideal, hybrid interior ideal) of $S$. For any $\left(\alpha_{1}, t_{1}\right),\left(\alpha_{2}, t_{2}\right) \in \operatorname{Im}\left(\tilde{f}_{\lambda}\right)$ with $\alpha_{1} \subset \alpha_{2}$ and $t_{1}>t_{2}$, we have $\tilde{f}_{\lambda}\left[\alpha_{2}, t_{2}\right] \subseteq \tilde{f}_{\lambda}\left[\alpha_{1}, t_{1}\right]$, where equality occurs if and only if there is no $x \in S$, such that $\alpha_{1} \subset \tilde{f}(x) \subseteq \alpha_{2}$ and $t_{2}<\lambda(x) \leqslant t_{1}$.

Proof. Let $\alpha_{1} \subset \alpha_{2}$ and $t_{1}>t_{2}$. Then $\tilde{f}_{\lambda}\left[\alpha_{2}, t_{2}\right] \subseteq \tilde{f}_{\lambda}\left[\alpha_{1}, t_{1}\right]$. Now, let $\tilde{f}_{\lambda}$ be a hybrid left ideal of $S$, such that $\tilde{f}_{\lambda}\left[\alpha_{1}, t_{1}\right]=\tilde{f}_{\lambda}\left[\alpha_{2}, t_{2}\right]$. Suppose that there exists an $x \in S$, such that $\alpha_{1} \subset \tilde{f}(x) \subseteq \alpha_{2}$ and $t_{2}<\lambda(x) \leqslant t_{1}$. Then $x \in \tilde{f}_{\lambda}\left[\alpha_{1}, t_{1}\right]$, but $x \notin \tilde{f}_{\lambda}\left[\alpha_{2}, t_{2}\right]$ : a contradiction. So, there exists no $x \in S$, such that $\alpha_{1} \subset \tilde{f}(x) \subseteq \alpha_{2}$ and $t_{2}<\lambda(x) \leqslant t_{1}$.

Conversely, suppose that $\tilde{f}_{\lambda}$ is a hybrid left ideal of $S$, such that there exists no $x \in S$ such that $\alpha_{1} \subset \tilde{f}(x) \subseteq \alpha_{2}$ and $t_{2}<\lambda(x) \leqslant t_{1}$. Since $\alpha_{1} \subset \alpha_{2}$ and $t_{2}<t_{1}$, we have $\tilde{f}_{\lambda}\left[\alpha_{2}, t_{2}\right] \subseteq \tilde{f}_{\lambda}\left[\alpha_{1}, t_{1}\right]$. Suppose that $\tilde{f}_{\lambda}\left[\alpha_{1}, t_{1}\right] \neq \tilde{f}_{\lambda}\left[\alpha_{2}, t_{2}\right]$. Then, there exists an $s \in S$, such that $s \in \tilde{f}_{\lambda}\left[\alpha_{1}, t_{1}\right]$ and $s \notin \tilde{f}_{\lambda}\left[\alpha_{2}, t_{2}\right]$. So, $\tilde{f}(s) \supseteq \alpha_{1}, \lambda(s) \leqslant t_{1}$ and $\tilde{f}(s) \subset \alpha_{2}$ or $\lambda(s)>t_{2}$, which implies $\alpha_{1} \subseteq \tilde{f}(s) \subset \alpha_{2}$ or $t_{2}<\lambda(s) \leqslant t_{1}$ : a contradiction. So, $\tilde{f}_{\lambda}\left[\alpha_{1}, t_{1}\right]=\tilde{f}_{\lambda}\left[\alpha_{2}, t_{2}\right]$.

Notation 2. Let $S$ and $T$ be a semigroup. A mapping $f: S \rightarrow T$ is said to be a homomorphism if $f(x y)=f(x) f(y)$ for all $x, y \in S$. In the sequel, Aut $(S)$ will denote the set of all automorphisms of $S$.

Definition 6. Let $S$ be a semigroup. An interior ideal $I$ of $S$ is called a characteristic interior ideal of $S$ if $h(I)=I$ for all $h \in A u t(S)$.

In [3], S. M. Hong and et al. have studied fuzzy interior ideals and fuzzy characteristic interior ideals of semigroups. We define the notion of a hybrid characteristic interior ideal of semigroup as follows:

Definition 7. Let $S$ be a semigroup. A hybrid interior ideal $\tilde{f}_{\lambda}$ of $S$ is called a hybrid characteristic interior ideal of $S$ if $\tilde{f}(h(x))=\tilde{f}(x)$ and $\lambda(h(x))=\lambda(x)$ for all $x \in S$ and all $h \in \operatorname{Aut}(S)$.

Theorem 8. Let $I$ be a non-empty subset of $S$. Then $I$ is a characteristic interior ideal of $S$ if and only if $\chi_{I}\left(\tilde{f}_{\lambda}\right)$ is a hybrid characteristic interior ideal of $S$.

Proof. Assume that $I$ is a characteristic interior ideal of $S$ and $x \in S$. Then, by Theorem $2, \chi_{I}\left(\tilde{f}_{\lambda}\right)$ is a hybrid interior ideal of $S$. 
If $x \in I$, then $\chi_{I}(\tilde{f})(x)=U$ and $\chi_{I}(\lambda)(x)=0$. Now, for any $h \in \operatorname{Aut}(S), h(x) \in h(I)=I$, which implies $\chi_{I}(\tilde{f})(h(x))=U=\chi_{I}(\tilde{f})(x)$ and $\chi_{I}(\lambda)(h(x))=0=\chi_{I}(\lambda)(x)$.

If $x \notin I$, then $\chi_{I}(\tilde{f})(x)=\phi$ and $\chi_{I}(\lambda)(x)=1$. Now, for any $h \in \operatorname{Aut}(S), h(x) \notin h(I)$, which implies $\chi_{I}(\tilde{f})(h(x))=\phi=\chi_{I}(\tilde{f})(x)$ and $\chi_{I}(\lambda)(h(x))=1=\chi_{I}(\lambda)(x)$. Thus, $\chi_{I}(\tilde{f})(h(x))=\chi_{I}(\tilde{f})(x)$ and $\chi_{I}(\lambda)(h(x))=\chi_{I}(\lambda)(x)$ for all $x \in S$ and, hence, $\chi_{I}\left(\tilde{f}_{\lambda}\right)$ is a hybrid characteristic interior ideal of $S$.

Conversely, assume that $\chi_{I}\left(\tilde{f}_{\lambda}\right)$ is a hybrid characteristic interior ideal of $S$. Then, by Theorem 2, $I$ is an interior ideal of $S$. Now, let $h \in \operatorname{Aut}(S)$ and $a \in I$. Then $\chi_{I}(\tilde{f})(a)=U$ and $\chi_{I}(\lambda)(a)=0$. Since $\chi_{I}\left(\tilde{f}_{\lambda}\right)$ is a hybrid characteristic interior ideal of $S$, we have $\chi_{I}(\tilde{f})(h(a))=\chi_{I}(\tilde{f})(a)$ and $\chi_{I}(\lambda)(h(a))=\chi_{I}(\lambda)(a)$ which imply $h(a) \in I$. So, $h(I) \subseteq I$ for all $h \in \operatorname{Aut}(S)$. Again, since $h \in \operatorname{Aut}(S)$ and $a \in I$, there exists a $b \in S$, such that $h(b)=a$.

Suppose that $b \notin I$. Then $\chi_{I}(\tilde{f})(b)=\phi$ and $\chi_{I}(\lambda)(b)=1$. Since $\chi_{I}(\tilde{f})(h(b))=\chi_{I}(\tilde{f})(b)$ and $\chi_{I} \lambda(h(b))=\chi_{I} \lambda(b)$, we have $h(b) \notin I$, i. e., $a \notin I$ : a contradiction. So, $b \in I$, i. e., $h(b) \in I$. Thus, $I \subseteq h(I)$ for all $h \in \operatorname{Aut}(S)$ and, hence, $I$ is a characteristic interior ideal.

Theorem 9. A non-empty hybrid structure $\tilde{f}_{\lambda}$ of $S$ is a hybrid characteristic interior ideal if and only if $[\alpha, t]-$ hybrid cut $\tilde{f}_{\lambda}[\alpha, t]$ of $S$ is a characteristic interior ideal of $S$ for all $\alpha \in \mathcal{P}(U)$ and $t \in[0,1]$, provided that $\tilde{f}_{\lambda}[\alpha, t] \neq \phi$.

Proof. Let $(\alpha, t) \in \operatorname{Im}\left(\tilde{f}_{\lambda}\right)$ for $\alpha \in \mathcal{P}(U)$ and $t \in[0,1]$. Then, by Theorem $5, \tilde{f}_{\lambda}[\alpha, t]$ is an interior ideal of $S$. Let $h \in \operatorname{Aut}(S)$ and $x \in \tilde{f}_{\lambda}[\alpha, t]$. Then $\tilde{f}(h(x))=\tilde{f}(x) \supseteq \alpha$ and $\lambda(h(x))=\lambda(x) \leqslant t$, which implies $h(x) \in \tilde{f}_{\lambda}[\alpha, t]$, and, so, $h\left(\tilde{f}_{\lambda}[\alpha, t]\right) \subseteq \tilde{f}_{\lambda}[\alpha, t]$. Now, let $x \in \tilde{f}_{\lambda}[\alpha, t]$ and $y \in S$ with $h(y)=x$. Then $\tilde{f}(y)=\tilde{f}(h(y))=\tilde{f}(x) \supseteq \alpha$ and $\lambda(y)=\lambda(h(y))=\lambda(x) \leqslant t$, which implies $y \in \tilde{f}_{\lambda}[\alpha, t]$. Since $x=h(y) \in$ $h\left(\tilde{f}_{\lambda}[\alpha, t]\right)$, we have $\tilde{f}_{\lambda}[\alpha, t] \subseteq h\left(\tilde{f}_{\lambda}[\alpha, t]\right)$. Thus, $\tilde{f}_{\lambda}[\alpha, t]$ is a characteristic interior ideal of $S$.

Conversely, assume that $\tilde{f}_{\lambda}[\alpha, t]$ is a characteristic interior ideal of $S$ for all $\alpha \in \mathcal{P}(U)$ and $t \in[0,1]$. Let $h \in A u t(S)$ and let $x \in S$ be such that $\tilde{f}(x)=\alpha$ and $\lambda(x)=t$. Then $x \in \tilde{f}_{\lambda}[\alpha, t]$. Since $h\left(\tilde{f}_{\lambda}[\alpha, t]\right)=\tilde{f}_{\lambda}[\alpha, t]$, we see that $h(x) \in \tilde{f}_{\lambda}[\alpha, t]$ implies $\tilde{f}(h(x)) \supseteq \alpha$ and $\lambda(h(x)) \leqslant t$. Now, let $\beta=\tilde{f}(h(x))$ and $s=\lambda(h(x))$. Then $h(x) \in \tilde{f}_{\lambda}[\beta, s]=h\left(\tilde{f}_{\lambda}[\beta, s]\right)$. Since $h$ is one-to-one, we have $x \in \tilde{f}_{\lambda}[\beta, s]$, which implies $\tilde{f}(x) \supseteq \beta$ and $\lambda(x) \leqslant s$, and, so, $\alpha \supseteq \beta$ and $t \leqslant s$. Thus, $\tilde{f}(h(x))=\beta=\alpha=\tilde{f}(x)$ 
and $\lambda(h(x))=s=t=\lambda(x)$, and, therefore, $\tilde{f}_{\lambda}$ is a hybrid characteristic interior ideal of $S$.

Recall that a semigroup $S$ is said to be left (resp., right) simple if it does not contain any proper left (resp., right) ideal of $S$. A semigroup $S$ is said to be simple if it does not contain any proper ideal of $S$. In [9], N. Kuroki introduced and studied some properties of fuzzy simple semigroups. We define the notion of hybrid simple semigroup as follows:

Definition 8. A semigroup $S$ is said to be hybrid simple if every hybrid ideal of $S$ is a constant function, i. e., for every hybrid ideal $\tilde{f}_{\lambda}$ of $S$, we have $\tilde{f}(x)=\tilde{f}(y)$ and $\lambda(x)=\lambda(y)$ for all $x, y \in S$.

Notation 3. If $S$ is a semigroup and $x \in S$, we define a subset, denoted by $I_{x}$, as follows:

$$
I_{x}:=\{y \in S \mid \tilde{f}(y) \supseteq \tilde{f}(x) \text { and } \lambda(y) \leqslant \lambda(x)\} .
$$

Proposition 1. If $\tilde{f}_{\lambda}$ is a hybrid right ideal (resp., hybrid left ideal, hybrid ideal) of $S$, then $I_{x}$ is a right ideal (resp., left ideal, ideal) of $S$ for every $x \in S$.

Proof. Let $x \in S$. Clearly, $\phi \neq I_{x} \subseteq S$. Let $y \in I_{x}$ and $t \in S$. Then $y t \in I_{x}$. Indeed: Since $\tilde{f}_{\lambda}$ is a hybrid right ideal of $S$ and $y, t \in S$, we have $\tilde{f}(y t) \supseteq \tilde{f}(y)$ and $\lambda(y t) \leqslant \lambda(y)$. Since $y \in I_{x}$, we have $\tilde{f}(y) \supseteq \tilde{f}(x)$ and $\lambda(y) \leqslant \lambda(x)$, and, so, $\tilde{f}(y t) \supseteq \tilde{f}(x)$ and $\lambda(y t) \leqslant \lambda(x)$, which imply $y t \in I_{x}$. Thus $I_{x}$ is a right ideal of $S$.

Corollary 1. [1, Corollary 3.6] For any nonempty subset $A$ of a semigroup $S$, the following are equivalent:

(i) $A$ is a two-sided ideal of $S$.

(ii) The characteristic hybrid structure $\chi_{A}\left(\tilde{f}_{\lambda}\right)$ in $S$ over $U$ is a hybrid two-sided ideal of $S$ over $U$.

Theorem 10. A semigroup $S$ is simple if and only if $S$ is hybrid simple.

Proof. Assume that $S$ is simple. Let $\tilde{f}_{\lambda}$ be a hybrid ideal of $S$ and $x, y \in S$. Then, by Proposition 1 , the set $I_{x}$ is an ideal of $S$. Since $S$ is simple, we have $I_{x}=S$. Since $y \in I_{x}$, we have $\tilde{f}(y) \supseteq \tilde{f}(x)$ and $\lambda(y) \leqslant \lambda(x)$. Similarly, we can get $\tilde{f}(x) \supseteq \tilde{f}(y)$ and $\lambda(x) \leqslant \lambda(y)$. So, $\tilde{f}_{\lambda}(y)=\tilde{f}_{\lambda}(x)$ and, therefore, $S$ is hybrid simple.

Conversely, assume that $I$ is an ideal of $S$. Then, by Corollary $1, \chi_{I}\left(\tilde{f}_{\lambda}\right)$ is a hybrid ideal of $S$. We now claim that $S=I$. Let $x \in S$. Since $S$ is 
hybrid simple, $\chi_{I}\left(\tilde{f}_{\lambda}\right)$ is a constant function, and $\chi_{I}\left(\tilde{f}_{\lambda}\right)(x)=\chi_{I}\left(\tilde{f}_{\lambda}\right)(y)$ for every $y \in S$. In particular, we have $\chi_{I}(\tilde{f})(x)=\chi_{I}(\tilde{f})(a)=U$ for any $a \in I$, which implies $x \in I$. Thus, $S \subseteq I$ and, hence, $S=I$.

Lemma. A semigroup $S$ is simple if and only if $S=S a S$ for every $a \in S$.

Proof. Assume that $S$ is simple and let $a \in S$. Then $S(S a S) \subseteq S a S$ and $(S a S) S \subseteq S a S$ imply $S a S$ is an ideal of $S$. Since $S$ is simple, we have $S a S=S$.

Conversely, let $A$ be an ideal of $S$ and let $a \in A$. Then $S=S a S$, $S a S \subseteq S A S \subseteq A$, which implies $A=S$. So $S$ is simple.

Theorem 11. Let $S$ be a semigroup. Then $S$ is simple if and only if every hybrid interior ideal of $S$ is a constant function.

Proof. Assume that $S$ is simple and $x, y \in S$. Let $\tilde{f}_{\lambda}$ be a hybrid interior ideal of $S$. Then, by the Lemma above we have $S=S x S=S y S$. Since $x \in S y S$, we have $x=a y b$ for $a, b \in S$. Since $\tilde{f}_{\lambda}$ is a hybrid interior ideal of $S$, we have $\tilde{f}(x)=\tilde{f}(a y b) \supseteq \tilde{f}(y)$ and $\lambda(x)=\lambda(a y b) \leqslant \lambda(y)$. Similarly, we can prove that $\tilde{f}(y) \supseteq \tilde{f}(x)$ and $\lambda(y) \leqslant \lambda(x)$ and, so, $\tilde{f}_{\lambda}$ is a constant function.

Conversely, assume that $\tilde{f}_{\lambda}$ is a hybrid ideal of $S$. Then $\tilde{f}_{\lambda}$ is a hybrid interior ideal of $S$. By the assumption, $\tilde{f}_{\lambda}$ is a constant function, and, so, $\tilde{f}_{\lambda}$ is hybrid simple. By Theorem 10, $S$ is simple.

Acknowledgement. The authors are grateful to the referees for their valuable comments and suggestions for improving the paper.

\section{References}

[1] Anis S., Khan M., Jun Y. B. Hybrid ideals in semigroups. Cogent Mathematics, 2017, vol. 4:1352117.

DOI: https://doi.org/10.1080/23311835.2017.1352117

[2] Hall T. E. On regular semigroups. J. Algebra, 1973, vol.24, no. 1, pp. 1-24. DOI: https://doi.org/10.1016/0021-8693(73)90150-6

[3] Hong S. M., Jun Y. B., Meng J. Fuzzy interior ideals in semigroups. Indian J. pure appl. Math., 1995, vol. 26 no. 9, pp. 859-863.

[4] Howie J. Fundamentals of semigroup theory, in: London Mathematical Society Monographs. New Series, Vol. 12, Oxford Science Publications, The Clarendon Press, Oxford University Press, New York, 1995.

[5] Kehayopulu N. On regular, intra-regular ordered semigroups. Pure Mathematics and Applications, 1993, vol. 4, no. 1, pp. 447-461. 
[6] Kehayopulu N., Tsingelis M. Fuzzy bi-ideals in ordered semigroups. Inform. Sci., 2005, vol. 171, pp. 13-28.

DOI: https://doi.org/10.1016/j.ins.2004.03.015

[7] Khan N. M., Davvaz B., Khan M. A. Ordered semigroups characterized in terms of generalized fuzzy ideals. Journal of Intelligent and Fuzzy Systems, 2017, vol. 32 no. 1 , pp. $1045-1057$.

DOI: https://doi.org/10.3233/JIFS-16339

[8] Kuroki N. On fuzzy ideals and fuzzy bi-ideals in semigroups. Fuzzy Sets and Systems, 1981, vol. 5, pp. 203-215.

[9] Kuroki N. Fuzzy semiprime ideals in semigroups. Fuzzy Sets and Systems, 1982, vol. 8, pp. $71-79$.

DOI: https://doi.org/10.1016/0165-0114(82)90031-8

[10] Kuroki N. On fuzzy semigroups. Inform. Sci., 1991, vol. 53, pp. 203-236.

DOI: https://doi.org/10.1016/0020-0255(91)90037-U

[11] Mordeson J. N., Malik D. S., Kuroki N. Regular semigroups. Fuzzy Semigroups, 2003, pp. 59-100.

[12] Rosenfeld A. Fuzzy groups. J. Math. Anal. Appl, 1971, vol. 35, pp. 512-517. DOI: https://doi.org/10.1016/0022-247X (71)90199-5

[13] Zadeh L. A. Fuzzy sets. Information and Control, 1965, vol. 8, pp. 338-353. DOI: https://doi.org/10.1016/S0019-9958(65)90241-X

Received April 0\%, 2019.

In revised form, October 25, 2019.

Accepted October 26, 2019.

Published online November 2, 2019.

Department of Mathematics,

Karunya Institute of Technology and Sciences,

Coimbatore - 641 114, India.

K. Porselvi

e-mail: porselvi94@yahoo.co.in

B. Elavarasan

belavarasan@gmail.com 